\title{
Spontaneous pneumothorax associated with COVID-19 pneumonia: a single-centre retrospective study
}

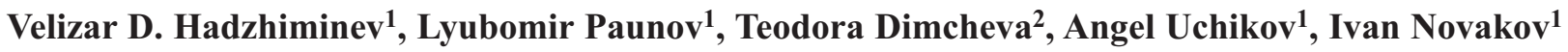 \\ ${ }^{1}$ Department of Special Surgery, Medical University, Plovdiv; ${ }^{2}$ Department of Medical Informatics, Biostatistics and \\ E-learning, Medical University, Plovdiv, Bulgaria
}

\begin{abstract}
Spontaneous pneumothorax (SP) is a rare complication of COVID-19 pneumonia; it affects both intubated and non-intubated patients. The pathogenesis includes barotrauma and pneumato-
\end{abstract}

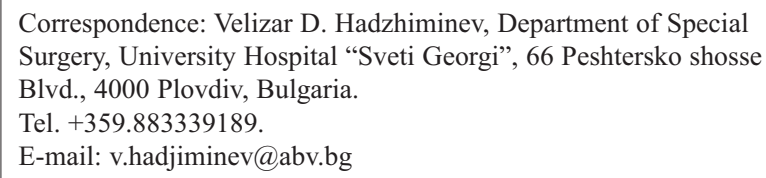

Contributions: VH, conceptualisation, methodology, formal analysis, investigation, writing - original draft, visualisation, project administration; LP, conceptualisation, formal analysis; TD, conceptualisation, methodology, statistical analysis, validation; AU, conceptualisation, formal analysis; IN, supervisor, conceptualization.

Conflict of interest: The authors declare that they have no competing interests, and all authors confirm accuracy.

Ethics approval and consent to participate: Due to the retrospective nature of the study, the IRB approval was not required, and all the data are de-identified. This study was conducted in accordance with Good Clinical Practice guidelines and ethical principles stated in the Declaration of Helsinki.

Informed consent: Before hospitalization, all patients provided their written informed consent for the further treatment and study participation.

Availability of data and materials: The datasets used and/or analyzed during the current study are available from the corresponding author on reasonable request.

Received for publication: 11 April 2021.

Accepted for publication: 2 August 2021.

${ }^{\circ}$ Copyright: the Author(s), 2021

Licensee PAGEPress, Italy

Monaldi Archives for Chest Disease 2022; 92:1884

doi: 10.4081/monaldi.2021.1884

This article is distributed under the terms of the Creative Commons Attribution Noncommercial License (by-nc 4.0) which permits any noncommercial use, distribution, and reproduction in any medium, provided the original author(s) and source are credited. cele formation. In the following article, we present case series of 18 patients with COVID-19 associated pneumothorax - a detailed demographic and clinical analysis were performed. The study revealed that men were more affected than women, especially above the age of 55 years; whilst, the distribution of intubated patients and those with spontaneous breathing were equal. Importantly, tube thoracostomy was the preferred method of treatment. The lethal outcome was observed in all patients on mechanical ventilation, due to the severe course of the underlying disease. The occurrence of pneumothorax in patients with COVID-19 is associated with poorer outcome of the disease, especially in those placed on mechanical ventilation.

\section{Introduction}

At the end of 2019, a new coronavirus serovar, SARS-CoV-2, which is associated with clusters of severe pneumonia was identified in Wuhan, China. On 11th February 2020, the World Health Organization (WHO) named the new coronavirus disease, COVID-19. A month later, a rapid increase in the number of infected individuals along with new outbreaks in various parts of the world declared COVID-19 a pandemic. By the end of 2020, there were approximately 79 million confirmed cases worldwide with over 1.5 million deaths $[1,2]$.

The principal lung presentation of COVID-19 is interstitial pneumonia, where one of its complications is spontaneous pneumothorax (SP). It is reported to be a rare event registered in less than $2 \%$ of patients with COVID-19 that require hospitalization $[3,4]$. The medical literature consists mainly of case reports [5-7]. The largest study about the current topic comprises 60 cases with COVID-19 and pneumothorax [8].

Here, we report the largest case series in Bulgaria of COVID19 related pneumothorax including non-intubated and intubated patients. Our study aims to analyze the demographic and clinical characteristics of a small cohort of patients with spontaneous pneumothorax associated with COVID-19 pneumonia.

\section{Materials and Methods}

This retrospective study included 18 patients with COVID-19 pneumonia and pneumothorax hospitalized in the University Hospital "Sveti Georgi", Plovdiv, Bulgaria for the period October to December 2020. Patients with COVID-19 and iatrogenic pneumothorax, due to central venous catheterization, were excluded. This study was conducted in accordance with Good Clinical 
Practice guidelines and ethical principles stated in the Declaration of Helsinki. We evaluated the patient demographic characteristics: the affected side, the size of the pneumothorax, the comorbidities and the breathing mode. The imaging method of choice was conventional chest radiography. We evaluated the treatment methods, complications and outcome. In addition, the average duration of the chest drainage and the average hospital stay were assessed. All statistical analyses were conducted in SPSS v.19 (SPSS, Chicago, IL, USA). Pearson's Chi-squared or Fisher's exact 2-tailed tests were used for the analysis of categorical data, while continuous variables were analyzed using Student's $t$-test; p-values $<0.05$ were considered to be statistically significant.

\section{Results}

\section{Demographic characteristics of the patients}

In our study, we included 18 patients with COVID-19 pneumonia and pneumothorax: 11 (61.1\%) were males and 7 (38.9\%) females. Pearson's Chi-square test showed that there were no statistically significant differences regarding the gender structure

Table 1. Demographic and clinical characteristics of the patients with pneumothorax and COVID-19.

Total (n)

\begin{tabular}{lcccc} 
Gender & & & & \\
Male & 11 & & & \\
Female & 7 & & & \\
Age group & & Male & Female & Total \\
$0-35$ years & & 1 & - & 1 \\
$36-55$ years & & 2 & - & 2 \\
$56-75$ years & & 5 & 5 & 10 \\
$>75$ years & & 3 & 2 & 5 \\
\hline
\end{tabular}

\section{Side affected}

Right

Left

Bilateral

\begin{tabular}{lc} 
Side affected & 10 \\
Right & 6 \\
Left & 2 \\
Bilateral & \\
Size & 3 \\
Small $<2 \mathrm{~cm}$ & 10 \\
Large $>2 \mathrm{~cm}$ & 2 \\
Total collapse & 3 \\
Tension pneumothorax & \\
\hline
\end{tabular}

Other comorbidities

No comorbidities 3

Arterial hypertension $\quad 12$

Atrial fibrillation

Diabetes mellitus

1

Breathing mode

Intubated and ventilated 9

Spontaneous

9

Management

Chest drain

VATS pleurodesis

17

Complications

Persistence of pneumothorax 5

None

13

Outcome

De-hospitalized 5

Death 13
( $p>0.05$, Chi-square $=0.89$ ). The registered mean age in the group was $67.83 \pm 3.5$ years. The patients were divided into four age groups. The first ( $0-35$ years) was presented by 1 male patient; the second group (36-55 years) consisted of 2 males and no females; the biggest group (56-75 years) had $5(50 \%)$ male and $5(50 \%)$ female patients. Finally, the last group (over 75 years) was presented by $3(60 \%)$ males and $2(40 \%)$ females. There were no statistically significant differences $(\mathrm{p}>0.05)$ in gender and age structures of the group (Table 1).

\section{Localization and size of the pneumothorax}

Pneumothorax affected the right side of 10 patients (55.6\%), the left side of 6 patients $(33.3 \%)$ and bilateral in 2 of the cases (11.1\%). Pearson's Chi-square test showed that there was no statistically significant difference $(\mathrm{p}>0.05$, Chi-square $=5.33$ ) regarding the localization. According to the size of the pneumothorax, we divided the patients into four groups measuring the distance between the visceral pleura of the collapsed lung and the thoracic wall at the level of the lung hilum (British Thoracic Society guidelines) [9]. For that purpose, we used conventional chest X-ray. Small pneumothorax $(<2 \mathrm{~cm})$ was registered in $3(16.7 \%)$ of the patients, whilst large pneumothorax $(>2 \mathrm{~cm})$ in $10(55.6 \%)$. A total collapse of the lung was observed in $2(11.1 \%)$ of the patients and $3(16.7 \%)$ had tension pneumothorax with shifted mediastinum. There was a statistically significant difference regarding the size of the pneumothorax in the group $(\mathrm{p}<0.05$, Chi-square=9.11). Most of the patients presented with large pneumothorax (Figure 1).

\section{Comorbidities and breathing mode}

Among the patients with COVID-19 and pneumothorax, 12 had arterial hypertension (66.7\%), 2 had diabetes mellitus (11.1\%), 1 had atrial fibrillation (5.6\%) and 3 had no other comorbidities $(16.7 \%)$. Pearson's Chi-square test showed a statistically significant difference $(p<0.05$, Chi-square $=17.11)$ regarding the comorbidities' distribution. Arterial hypertension was predominant in the group.

The patients aged between 56-75 years were the most affected, followed by those aged over 75 years. All of them had other comorbidities.

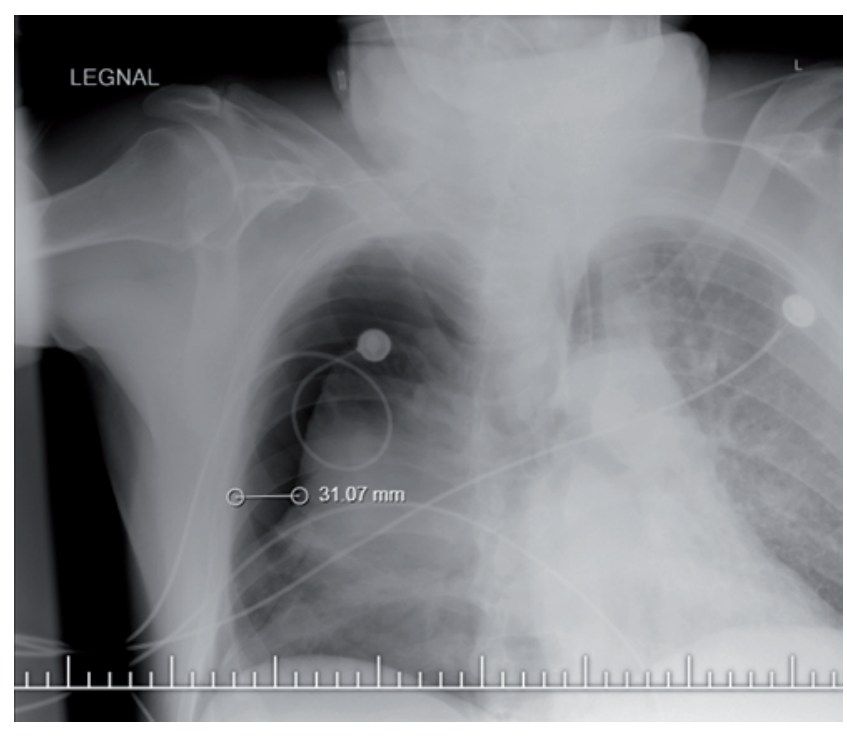

Figure 1. Large right-sided pneumothorax $(>2 \mathrm{~cm})$ in a patient with COVID-19. 
$9(50 \%)$ of the patients were with spontaneous breathing, 8 were males and only 1 was female. $9(50 \%)$ were intubated and ventilated (including the tracheostomised): 6 females and 3 males. All of them were placed on pressure-controlled ventilation (peak pressure $<30 \mathrm{~cm} \mathrm{H}_{2} \mathrm{O}, \mathrm{PEEP}>10 \mathrm{~cm} \mathrm{H}_{2} \mathrm{O}$ ). Our case series suggested that most of the female patients with COVID-19 and pneumothorax were on mechanical ventilation.

\section{Management, complications and outcome}

In the present study, tube thoracostomy was performed in 17 patients $(94.4 \%)$ and video-assisted thoracoscopic surgery with pleurodesis in $1(5.6 \%)$. The persistence of pneumothorax as a complication was observed in 5 patients $(27.8 \%)$, all of whom were on mechanical ventilation. In these cases, the insertion of additional chest tube was performed. The lethal outcome was registered in 13 patients $(72.2 \%)-6$ men and 7 women. 5 of the patients (all men) were discharged. The highest mortality rate was registered in the age groups 56-75 years (7 cases) and over 75 years ( 5 cases). The Pearson's test showed that there was no statistically significant difference in the patients' age distribution regarding the disease outcome $(\mathrm{p}>0.05$, Chi-square $=3.56)$.

The mean duration of the thoracic drainage in the group was 7.3 days. The registered mean hospital stay was 11.1 days.

\section{Discussion}

Pneumothorax is characterized as a serious but relatively rare complication in patients with COVID-19. It has been reported in $1 \%$ of the hospitalized patients, in $2 \%$ of those in the ICU, and in $1 \%$ of those who have died from the infection. Chen et al. described only 1 case out of 99 confirmed patients, whilst Yang et al. found only one with pneumothorax out of 92 autopsied patients $[3,10,11]$.

The major pathogenetic mechanism of pneumothorax in patients with COVID-19 is due to diffuse alveolar damage caused by the virus itself. It leads to alveolar rupture followed by interstitial emphysema and the formation of pneumatocele. Multinucleated syncytial cells and atypically enlarged pneumocytes are observed in the alveolar spaces as a result of the viral cytopathic effect. Pneumocyte desquamation and formation of hyaline membranes that are associated with acute respiratory distress syndrome may also be detected. Rupture of the pneumatocele leads to pneumothorax. This can occur in both non-intubated patients due to severe cough as a possible cause. In addition, those on pressure-controlled mechanical ventilation as a result of the positive pressure barotrauma [12-15].

A detailed study of COVID-19 associated pneumothorax was performed by Martinelli et al. [8] who followed up 60 patients. They found that it was 3 times more common in men and mainly right-sided. In addition, it was more common after the age of 50 . Both patients on mechanical ventilation and those with spontaneous breathing were almost equally affected, but the survival rate in the first group was lower. As a therapeutic method of choice, the authors suggested the placement of a chest drain [8].

In our study, we present the largest case series of pneumothoraxes associated with COVID-19 in Bulgaria. All data were collected for the period October-December 2020; during that time, Bulgaria registered the year's highest infection rate. In our group, males were more affected than females, which is consistent with the demographics of pneumothorax. Moreover, there was a severe course of COVID-19 in men. SP was more common in the age group 56-75 years; these patients were more likely to have another underlying lung disease. It was noted that the right side was more affected and that most cases were presented with large $(>2 \mathrm{~cm})$ pneumothorax.

The principal therapeutic method of choice in more than $90 \%$ of our patients was tube thoracostomy. It was technically easy to perform, even at the patient's bedside. This was of particular value, especially in patients placed on mechanical ventilation. In 5 of the ventilated patients, the persistence of pneumothorax was observed on the control X-ray $24 \mathrm{~h}$ after the initial drainage, which was explained by the persistent alveolar permeability and active inflammation of COVID-19. Unfortunately, lethal outcome was observed in all intubated patients and was primarily because of the severe course of the underlying lung disease. The probable mechanism of pneumothorax in these cases was barotrauma, as well as the diffuse alveolar damage from the virus which led to the formation of interstitial emphysema and pneumatocele. The data in our study were similar to those reported by Martinelli et al. [8]. The design of our study does not include follow-up of the discharged patients.

The outcome in patients with COVID-19 pneumonia and pneumothorax reported in our study as well as other authors was dependent on the severity of the underlying lung injury and the impaired gas exchange. High-volume surgical interventions are recommended for this to be avoided. Thus, the placement of a chest drain should be a therapeutic method of choice, even if it is for a longer period (15-20 days) [16-20].

\section{Conclusions}

We investigated the demographic and clinical features of a small cohort of patients with pneumothorax associated with COVID-19 pneumonia. Our results suggested that the occurrence of pneumothorax is a serious complication, which is related to a poorer outcome of the disease and a high mortality rate in ventilated patients. Further investigations on that problem are warranted.

\section{References}

1. Zhang G, Zhang J, Wang B, et al. Analysis of clinical characteristics and laboratory findings of 95 cases of 2019 novel coronavirus pneumonia in Wuhan, China: a retrospective analysis. Respir Res 2020;21:74.

2. WHO. COVID-19 Weekly Epidemiological Update. December 2020.

3. Chen N, Zhou M, Dong X, et al. Epidemiological and clinical characteristics of 99 cases of 2019 novel coronavirus pneumonia in Wuhan, China: a descriptive study. Lancet 2020;395:507-13.

4. Wang D, Hu B, Hu C, et al. Clinical characteristics of 138 hospitalized patients with 2019 novelcoronavirus-infected pneumonia in Wuhan, China. JAMA 2020;323:1061-9.

5. Hameed M, Jamal W, Yousaf M, et al. Pneumothorax in Covid19 pneumonia: A case series. Respir Med Case Reports 2020;31:101265.

6. Hollingshead C, Hanrahan J. Spontaneous pneumothorax following COVID-19 pneumonia. IDCases 2020;21:e00868.

7. Ferreira JG, Rapparini C, Moreno Gomes B, et al. Pneumothorax as a late complication of COVID-19. Rev Inst Med Trop S Paulo 2020;62:5-9. 
8. Martinelli AW, Ingle T, Newman J, et al. COVID-19 and pneumothorax: a multicentre retrospective case series. Eur Respir J 2020;56:2002697.

9. MacDuff A, Arnold A, Harvey J. Management of spontaneous pneumothorax: British Thoracic Society pleural disease guideline 2010. Thorax 2010;65:ii18-31.

10. Yang F, Shi S, Zhu J, et al. Analysis of 92 deceased patients with Covid-19. J Med Virol 2020;92:2511-15.

11. Nicola M, Neill NO, Sohrabi C, et al. Evidence based management guideline for the COVID-19 pandemic - Review article. Int J Surg 2020;77:206-16.

12. Murayama S, Gibo S. Spontaneous pneumomediastinum and Macklin effect: Overview and appearance on computed tomography. World J Radiol 2014;6:850-4.

13. Xu Z, Shi L, Wang Y, Zhang J, et al. Pathological findings of COVID-19 associated with acute respiratory distress syndrome. Lancet Respir Med 2020;8:420-2.

14. Sihoe ADL, Wong RHL, Lee ATH, et al. Severe acute respira- tory syndrome complicated by spontaneous pneumothorax. Chest 2004;125:2345-51.

15. Bernheim A, Mei X, Huang M, et al. Chest CT findings in coronavirus disease-19 (COVID-19): relationship to duration of infection. Radiology 2020;295:685- 91.

16. Li L, Li R, Wu Z, et al. Therapeutic strategies for critically ill patients with COVID-19. Ann Intensive Care 2020;10:45.

17. McCool FD. Diseases of the diaphragm, chest wall, pleura and mediastinum. In: Goldman L, Schafer AI, editors. GoldmanCecil medicine. 26th ed. Philadelphia: Elsevier; 2020. p. 602-12.

18. Mallick T, Dinesh A, Engdahl R, Sabado M. COVID-19 complicated by spontaneous pneumothorax. Cureus 2020;12:e9104.

19. Rohailla S, Ahmed N, Gough K. SARS-CoV-2 infection associated with spontaneous pneumothorax. CMAJ 2020;192:e510.

20. Ucpinar BA, Sahin C, Yanc U. Spontaneous pneumothorax and subcutaneous emphysema in COVID-19 patient: case report. J Infect Public Health 2020;13:887-9. 